\title{
DETERMINATION OF ABSOLUTE PROPER MOTIONS BY USE OF AUTOMATED MEASUREMENTS OF TAUTENBURG PLATES
}

\author{
R.-D. SCHOLZ \\ Central Institute of Astrophysics \\ DDR - 1561 Potsdam \\ Germany, Dem. Rep.
}

\begin{abstract}
From measurements of Tautenburg Schmidt plates with the APM in Cambridge positional accuracies per plate of 0.05 for stars and of 0 ". 10 for galaxies were achieved. With $0.3 / 100 a$ accuracy in a single stellar proper motion we obtained the absolute proper motion of the M3 globular cluster in good agreement between the two pairs of plates used.
\end{abstract}

\section{Introduction}

Proper motions of stars obtained with respect to distant galaxies are of great importance for the implementation of a nonrotating coordinate system. In the Potsdam program of determining absolute proper motions in selected fields of the Northern sky Tautenburg Schmidt plates with epoch differences of more than 20 years are used. This program is part of international efforts being made to connect existing reference frames like FK5 and forthcoming reference frames of space astrometry with an extragalactic reference frame. For a description of the program see Dick et al. (1987) and Yatsenko et al. (1987).

The development of high speed scanning microdensitometers for accurate coordinate measurements of the images of all objects on a Schmidt plate has brought some progress in proper motion work, especially concerning the tangential motions of groups of objects against the background of large numbers of galaxies. Kibblewhite $e t$ al. (1982) reported on very promising first results of proper motion studies from measurements on Palomar and UK Schmidt plates with the Automated Photographic Measuring (APM) facility at Cambridge/UK. This work is now being continued by Evans (1988). Owing to the assistance of Prof. Argue and the APM group in Cambridge it was possible to measure some Tautenburg Schmidt plates on the APM. First results of the reduction of these measurements are briefly described here.

\section{Results}

In each of 4 fields (one with the M3 globular cluster in the centre, one with M3 in the plate comer and two fields including parts of he Virgo galaxy cluster) two pairs of plates were measured. The 
$24 \times 24 \mathrm{~cm}^{2}$ plate area corresponds to $3.2 \times 3.2$ square degrees. The number of objects measured on one plate varied from 16000 to 70000 .

Prior to the determination of proper motions we compared plates of one epoch and eliminated most of noise images. The positional accuracy with different magnitude classes of stars and galaxies was investigated in plate-to-plate solutions. In 7 of 8 cases an accuracy from 0.8 to 1.5 microns (0.04 to 0.08) was obtained for faint stars. For galaxies we achieved a positional accuracy of 1.5 to 2.0 microns ( 0.08 to 0 ".10) except the second epoch M3 plates with only 4 microns (0"2). For the determination of proper motions we used complete third and second order polynomials, a linear model and the best model of stepwise regression (see Hirte et al. 1989). An iterative procedure considering larger coordinate shifts for brighter stars was used in the plate matching of different epochs. For the first time we obtained an accuracy of $0.3 / 100 a$ for faint stars, whereas the accuracy for AGK3 stars was comparable with former results (e.g. Scholz and Rybka 1988).

Webbink (1988) stressed the need of determining absolute proper motions of globular clusters directly with respect to galaxies. For the most part former investigations have been relative proper motion studies affected by serious uncertainties in the reduction to absolute proper motions. Tucholke et al. (1988) used stars from the Lick program with known absolute proper motions as reference stars and obtained mean cluster motions with an error of 0.05 to 0"10/100a. Our preliminary results in an attempt to derive the absolute proper motion of the M3 cluster are described here.

All stars within a circle of 8.2 arc minutes radius around the cluster centre were taken as M3 stars. In order to minimize possible magnitude effects only one magnitude interval with 790 reference galaxies and $680 \mathrm{M} 3$ stars was selected. The mean proper motion of the cluster from two pairs of plates was obtained as $0.11 / 100 a$ in $\mathrm{x}$ and $-0.20 / 100 a$ in $\mathrm{y}$. The variation in the mean cluster motion with different reduction models was less than 0.02/100a. The difference of the cluster motion between the two pairs of plates was about 0.05/100a. Using more pairs of plates available in the Tautenburg plate archive we could minimize possible systematic effects.

\section{References}

Dick,W.R., Ruben,G., Schilbach,E., Scholz,R.-D. (1987) Astron. Nachr. 308, 211-216.

Evans,D.W. (1988), Thesis, Cambridge.

Hirte,S., Dick,W.R., Schilbach,E., Scholz,R.-D. (1989) In: Errors, bias and uncertainties in astronomy, Proc. IAU Colloq. Strasbourg, in press.

Kibblewhite,E.J., Irwin,M.J., Bridgeland,M.T., Bunclark,P.S. (1982), Occ. Rep. R. Obs. Edinburgh 10, 79-89.

Scholz,R.-D., Rybka,S.P. (1988), Astron. Nachr. 309, 47-52.

Tucholke,H.-J., Brosche,P., Geffert,M. (1988) in: J.E.Grindlay, A.G.Davis Philip (eds.), The Harlow-Shapley Symposium on Globular Cluster Systems in Galaxies, IAU, 525-526.

Webbink,R.F. (1988) ibidem, 49-60.

Yatsenko,A.I., Rybka,S.P., Scholz,R.-D. (1987) Astron. Nachr. 308, 319. 